\title{
Clinical effects and economical impact of dutasteride and finasteride therapy in Italian men with LUTS
}

\author{
Luca Cindolo $^{1}$, Francesco Berardinelli ${ }^{1}$, Caterina Fanizza ${ }^{2}$, Marilena Romero ${ }^{2}$, \\ Luisella Pirozzi $^{2}$, Fabiola Raffaella Tamburro ${ }^{1}$, Fabrizio Pellegrini ${ }^{1}$, Fabio Neri ${ }^{1}$, \\ Andrea Pitrelli ${ }^{3}$, Luigi Schips ${ }^{1}$ \\ ${ }^{1}$ S. Pio da Pietrelcina Hospital, Dept. of Urology, Vasto, Italy; \\ ${ }^{2}$ Department of Clinical Pharmacology and Epidemiology, Consorzio Mario Negri Sud, Santa Maria Imbaro, Italy; \\ ${ }^{3}$ Access to Medicine, GlaxoSmithKline spa, Verona, Italy.
}

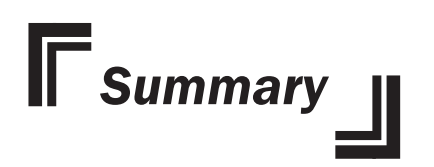

Objectives: To investigate differences in the risk of benign prostatic hyperplasia (BPH)related hospitalization, for surgical and non-surgical reasons, and of new prostate cancer (PCa) diagnosis between patients under dutasteride or finasteride treatment.

Material and methods: A retrospective cohort study was conducted using data from record-linkage of administrative databases. Men aged $\geq 40$ years old who had received a prescription for at least 10 boxes/year (index years: 2004-06) were included. The association of the outcomes was assessed using a multiple Cox proportional hazard model. Propensity scorematched analysis and a 5-to-1, greedy 1:1 matching algorithm were performed. The budget impact analysis of dutasteride vs finasteride in BPH-treated patient was performed.

Results: From an initial cohort of about 1.5 million of Italian men, 19620 were selected. The overall hospitalization for BPH-non surgical reasons, for BPH-related surgery and for new detection of PCa incidence rates (IRs) were 8.20 (95\% CI, 7.62-8.23), 18.0 (95\% CI, 17.12-18.93) and 8.62 (95\% CI, 8.03-9.26) per 1000 person-years, respectively. The multivariate analysis after the propensity score-matching showed that dutasteride was associated with an independent reduced likelihood of hospitalization for BPH-related surgery (HR 0.82; 95\% CI 0.73-0.93; $p=0.0025$ ) and of newly detected PCa (HR: 0.76,95\% CI, 0.65-0.85; $p=0.0116)$. The IR for BPH-non surgical reasons was 8.07 (95\% CI, 7.10-9.17) and 9.25 (95\% CI, 8.19-10.44) per 1000 person-years, respectively. The IR for BPH-related surgery was 18.28 (95\% CI, 17.17-20.32) and 21.28 (95\% CI, 19.24-23.06) per 1000 person-years among patients under dutasteride compared with those under finasteride, respectively. For new-onset PCa, the IR was 8.01 (95\% CI, 7.07-9.08) and 9.38 (95\% CI, 8.32-10.58) per 1000 person-years The pharmacoeconomical evaluation showed that the net budget impact of the use of dutasteride vs. finasteride in $1000 \mathrm{BPH}$-treated patient for 1 year induces a saving of $3933 €$.

Conclusions: The clinical effects of dutasteride and finasteride are slightly different. The likelihood of hospitalization for BPH-related surgery and of newly detected PCa seems to be in favor of dutasteride. The budget impact analyses showed a slightly benefit for dutasteride. Comparative prospective studies are necessary to confirm these results.

KEY WORDS: Benign prostatic hyperplasia (BPH); Dutasteride; Finasteride; Epidemiology; Medical record-linkage.

Submitted 14 April 2013; Accepted 5 October 2013

\section{INTRODUCTION}

Lower urinary tract symptoms (LUTS) are common in aging men with a prevalence ranges from 10.3 to $25.1 \%$ depending on the severity threshold (1). Benign prostatic hyperplasia (BPH) and benign prostatic enlargement
(BPE) have been recognized as the major contributing factors to the development of LUTS. The first-line pharmacological therapy for moderate-to-severe non-neurogenic male LUTS includes alpha-adrenoreceptor antago- 
nists (ABs) and 5alpha-reductase inhibitors (5ARIs) alone or in combination (2). ABs induce a rapid symptom relief, while the 5ARIs modify the BPH natural history by delaying the disease progression (3-6). Finasteride and dutasteride are the two 5ARIs: finasteride inhibits the 5-alpha-reductase isoenzyme type 2, whereas dutasteride inhibits both isoenzyme 1 and 2 . The clinical value of the greater serum dihydrotestosterone suppression achieved by dutasteride (7) remains unclear (2). Nowadays due to the limited literature (811) the question of "what is the best 5ARI" remains unanswered. Another point of uncertainty is about the economic impact of the use of dutasteride instead of finasteride. In an attempt to clarify these aspects, we previously performed an observational study on an unselected population that showed a reduction in BPH-related hospitalization risk in dutasteride- compared to finasteride-treated patients (12). In that paper (12) we also dealt with the hard issue of the detection of prostate cancer (PCa) under 5ARIs treatments (13-16) showing a positive trend in favor of dutasteride.

Herein, we report the new results of extended analysis investigating the clinical and economic differences between dutasteride and finasteride treatment in an Italian male population $\geq 40$ years with LUTS.

\section{Material AND MEthodS}

A retrospective study was conducted based on information from three databases: Italian population registry, pharmaceutical prescription data, and hospital discharge record including information on about 1.500 .000 male aged $\geq 40$ years from 22 Local Health Units from Northern and Southern Italy for 6 consecutive years (January 1st 2004 and December 31st 2009).

\section{Data sources}

The Italian population registry provide demographic information (date of birth, sex and date of death if this occurred) on each subject. The pharmaceutical prescription database records all prescriptions reimbursed by the NHS (drugs coded according to the international Anatomical Therapeutic Chemical system - ATC) (17).

The hospital records include detailed information on primary diagnosis and up to five coexisting diagnoses, performed procedures, and admission/discharge dates. The diagnoses were classified according to the International Classification of Diseases-Ninth Revision, Clinical Modification (ICD9-CM) (18). A record linkage of these three databases was carried out and pharmacological and clinical history for each patients was obtained. The reliability of this strategy to produce an epidemiological survey has been previously validated and reported (19-21). All security and protection measures for patient's data was performed according to National laws on privacy protection.

\section{Patients and Drugs}

The cohort consisted of men aged $\geq 40$ years, who received prescription for at least 10 boxes/year of finasteride or dutasteride between 1 January 2004 and 31 December 2006 (index years). The first prescription of one of these drugs during the index years was considered as index date (Day 0). The exclusion criteria were either ABs monotherapy and/or short-term 5-ARI therapy $(<10$ boxes/year). For all patients, the databases were searched during the 12-months period preceding the index date to verify the absence of $\mathrm{BPH}$-complications and $\mathrm{PCa}$. Specifically, patients with an urethral stricture (ICD9CM: 598, 589.0, 598.00, 598.01, 598.1, 598.2, 598.8, 598.9) and/or with PCa diagnoses (ICD9-CM: 185, $198.82,233.4,236.5,239.5, \mathrm{~V} 10.46)$ and/or at least a prescription of LHRH analogues and/or antiandrogens, were not considered eligible. Patients using ABs (alfuzosin, tamsulosin, terazosin) were included in the study. Patients with acute or chronic urinary retention secondary to BPH and treated at the emergency department without hospital admission were not considered. Moreover, to assess the comorbidities, the Charlson Comorbidity Index (CCI) with the Dartmouth-Manitoba modification was used (22).

\section{Clinical outcomes}

Follow-up for each identified patient is extended from the index date to five years or until the occurrence of the following major events: 1) hospitalization for BPH-non surgical reasons); 2) hospitalization for BPH-related surgery; 3) new diagnosis of PCa.

BPH-related hospitalization was considered when the hospital records included primary diagnosis and/or procedures related to $\mathrm{BPH}$.

The presence of the ICD9-CM 600.xx (prostate hyperplasia) and 222.2 (benign prostate tumor) codes as primary diagnosis without surgical procedures was considered hospitalization for "BPH-non surgical reasons". The presence of ICD9-CM 57.0, 57.91, 57.92, 60.21, 60.29, 60.3, 60.4 codes (open or transurethral resection/ablation of prostate or bladder neck), as primary or secondary surgical procedures with any primary diagnoses, was considered hospitalization for "BPH-related surgery".

The new diagnosis of $\mathrm{PCa}$ was identified through hospitalization (ICD9-CM:185, 198.82, 233.4, 236.5, 239.5, V10.46) and/or PCa medical therapy (Gonadotripins releasing hormones agonists L02AE01, L02AE02, L02AE03, LO2AE04; and/or antiandrogens: L02BBO1, L02BBO2, LO2BB03).

\section{Analysis of health resources utilization}

The budget impact analysis of dutasteride vs. finasteride in BPH-treated patient according to the Italian NHS perspective has been performed starting form an hypothetical cohort of 1000 BPH-treated men under finasteride for one year, here and after "current scenario"; in our analysis this hypothetic cohort has been fully switched to dutasteride, here and after "alternative scenario".

The incidence rates for 1000 person-years by outcomes after propensity score matching were used as source for the budget impact analysis model. Drug consumption has been calculated assuming an annual $80 \%$ compliance to both treatment (300 days of therapy); in both scenarios patients undergoing to BPH-related surgery withdrawn from treatment (assuming they don't need further treatment for BPH). The health resources utilization in both scenarios has been calculated starting from the inci- 
dence rates (both surgical and non surgical reasons) for 1000 persons/years after propensity score matching. Hospital records have been used to estimate the average hospitalization costs according to NHS perspective. The impact on NHS annual budget related to variation of PCa detection rate observed with dutasteride vs. finasteride was not analyzed.

\section{Statistical analysis}

For the whole sample, patients' characteristics were reported as frequency (percentage) and mean \pm standard deviation. Differences between patients' treatment subgroups were assessed using standardized difference. For major outcomes, crude incidence rates (IRs) per 1000 men-year were calculated as the number of events divided by the number of person-years of follow-up.

Furthermore, to check consistency of our results, a propensity score (PS)-matched analysis was performed (24-25). A logistic model -including the same covariates used in the multivariate Cox model, plus quadratic terms and a set of two-term interactions between the same covariates- was performed to predict the probability to be assigned to study drugs. PS logistic model was selected in a stepwise fashion and pair-wise comparisons were performed. A 5-to-1, greedy 1:1 matching algorithm (26) was used to identify a unique matched control for treated patient according to their PS. Adequacy of covariate balance in the matched sample was assessed via standardized difference between the two groups, considering differences less than $10 \%$ as good balance (27).

The association of hospitalization for BPH, BPH-related surgery, PCa was assessed using a multiple Cox proportional hazard model. All multivariate analyses were adjusted for the following variables: age, Charlson comorbidity score, previous hospitalization for $\mathrm{BPH}$, previ- ous BPH-related surgery, pre-existing severity factors, previous pharmacological treatment with ABs. Results are expressed as hazard ratios (HRs) and 95\% confidence intervals (CIs).

P-values $<0.05$ were considered significant. All analyses were performed using SAS Statistical Package Release 9.2 (SAS Institute, Cary, NC, USA).

\section{Results}

\section{Patients characteristics}

From 1.417.969 men aged $\geq 40$ years, 19620 were chronically exposed to 5ARIs; 13195 received finasteride and 6425 dutasteride. No significant differences were observed between these two groups with exception of previous ABs therapy (Table 1).

\section{Clinical outcomes during follow-up}

During 5 years, 841 patients were hospitalized for BPHnon surgical reasons, 2006 for BPH-related surgery and 749 were newly diagnosed with PCa. The overall hospitalization IR for BPH-non surgical reasons and for BPH-related surgery were 8.20 (95\% CI, 7.62-8.23) and 18.0 (95\% CI, 17.12-18.93) per 1000 person-years, respectively.

The matched analysis identified 6362 men under dutasteride that were matched with a similar cohort under finasteride, without significant differences between groups (Table 2).

Among patients under dutasteride compared with those under finasteride the IR for BPH-non surgical reasons was 8.07 (95\% CI, 7.10-9.17) and 9.25 (95\% CI, 8.19-10.44) per 1000 person-years, respectively. Moreover, the IR for BPH-related surgery was 18.28 (95\% CI, 17.17-20.32) and 21.28 (95\% CI, 19.24-23.06) per 1000 person-years

Table 1.

Patients' characteristics according to drug used (finasteride or dutasteride).

\begin{tabular}{|l|c|c|c|}
\hline Variable & $\begin{array}{c}\text { Finasteride (13195 pz) } \\
\mathbf{N}(\%)\end{array}$ & $\begin{array}{c}\text { Dutasteride (6425 pz) } \\
\mathbf{N}(\%)\end{array}$ & $\begin{array}{c}\text { Standardized } \\
\text { difference (\%) }\end{array}$ \\
\hline Mean Age (mean \pm SD) & $72.25(9.14)$ & $71.62(8.46$ & -7.1538 \\
\hline Age & & & -5.9106 \\
\hline $50-55$ & $509(3.86)$ & $178(2.80)$ & 8.8940 \\
\hline $66-65$ & $4917(37.26)$ & $2647(41.61)$ & 1.7876 \\
\hline $76-85$ & $5254(39.82)$ & $2589(40.69)$ & -11.0948 \\
\hline Charlson score & $2515(19.06)$ & $948(14.90)$ & 1.4657 \\
\hline $1-2$ & $10945(82.95)$ & $5312(83.50)$ & 0.6326 \\
\hline Previous hospitalization for BPH (non surgical reasons) & $1397(10.59)$ & $686(10.78)$ & -3.1069 \\
\hline Previous hospitalization for BPH-related surgery & $853(6.46)$ & $364(5.72)$ & 5.1632 \\
\hline Previous BPH complications (severity factors) & $924(7.00)$ & $533(8.38)$ & 3.2896 \\
\hline Previous alphablockers therapy & $59(0.30)$ & $32(0.50)$ & -0.7011 \\
\hline
\end{tabular}

* Standardized difference greater than $10 \%$ represents meaningful imbalance in explored variables between treatment groups. 
Table 2.

Patients' characteristics according to drug used (finasteride or dutasteride) after propensity score matching.

\begin{tabular}{|c|c|c|c|}
\hline Variable & $\begin{array}{c}\text { Finasteride (6362 pz) } \\
\mathrm{N}(\%) \\
\end{array}$ & $\begin{array}{c}\text { Dutasteride (6362 pz) } \\
\text { N (\%) } \\
\end{array}$ & $\begin{array}{r}\text { Standardized } \\
\text { difference (\%) }\end{array}$ \\
\hline Mean Age (mean \pm SD) & $71.68(8.42)$ & $71.62(8.46)$ & 0.71092 \\
\hline \multicolumn{4}{|l|}{ Age } \\
\hline 40-55 & $175(2.75)$ & $178(2.80)$ & 0.28712 \\
\hline $56-65$ & $2641(41.51)$ & $2647(41.61)$ & 0.19137 \\
\hline $66-75$ & 2589 (40.69) & 2589 (40.69) & 0.00000 \\
\hline $76-85$ & $957(15.04)$ & $948(14.90)$ & -0.39649 \\
\hline \multicolumn{4}{|l|}{ Charlson score } \\
\hline$\underline{0}$ & $5294(83.21)$ & $5312(83.50)$ & 0.75957 \\
\hline $1-2$ & $695(10.92)$ & $686(10.78)$ & -0.45479 \\
\hline$>=3$ & $373(5.86)$ & $364(5.72)$ & -0.6056 \\
\hline Previous hospitalization for BPH (non surgical reasons) & $528(8.30)$ & $533(8.38)$ & 0.28427 \\
\hline Previous hospitalization for BPH-related surgery & $19(0.30)$ & $32(0.50)$ & 3.23449 \\
\hline Previous BPH complications (severity factors) & $292(4.59)$ & $272(4.28)$ & -1.52745 \\
\hline Previous alphablockers therapy & $3890(61.14)$ & $3893(61.19)$ & 0.09675 \\
\hline
\end{tabular}

* Standardized difference greater than $10 \%$ represents meaningful imbalance in explored variables between treatment groups.

among patients under dutasteride compared with those under finasteride, respectively. For new-onset PCa, the IR was 8.01 (95\% CI, 7.07-9.08) and 9.38 (95\% CI, 8.3210.58) per 1000 person-years (Table 3 ).

The multivariate analysis after the propensity score matching Cox model showed that dutasteride was associated with an independent reduced likelihood of hospitalization for BPH-related surgery (HR 0.82; 95\% CI $0.73-0.93 ; p=0.0025)$ and of newly detected PCa (HR: 0.76, 95\% CI, 0.65-0.85; p = 0.0116) (Table 4).
Annual budget impact analysis

In the "current scenario" an hypothetical cohort of 1000 BPH-treated patient for 1 year with finasteride generates a total annual impact on NHS budget of 1.017.444 €: $13,4 \%$ of this cost is related to finasteride cost (136.145 €), 66,4\% is related to hospitalizations due to BPH-related surgery $(675.423 €)$ and $20,2 \%$ is related to hospitalizations for BPH-non surgical reasons (205.872 €).

In the "alternative scenario" is generated a total annual

Table 3.

Incidence rate for 1000 person-years by outcome considered in finasteride and dutasteride groups after propensity score matching.

\begin{tabular}{|l|cc|cc|}
\hline Outcome & \multicolumn{2}{|c|}{ Finasteride } & \multicolumn{2}{c|}{ Dutasteride } \\
& Incidence rate & $\mathbf{9 5 \%} \mathbf{~ l ~}$ & Incidence rate & 95\% Cl \\
\hline Hospitalization for BPH (non surgical reasons) & 9.25 & $8.19-10.44$ & 8.07 & $7.10-9.17$ \\
\hline Hospitalization for BPH-related surgery & 21.28 & $19.24-23.06$ & 18.28 & $17.17-20.32$ \\
\hline Newly detected prostate cancer & 9.38 & $8.32-10.58$ & 8.01 & $7.07-9.08$ \\
\hline
\end{tabular}

Table 4.

Results of propensity score matching Cox model: dutasteride vs. finasteride.

\begin{tabular}{|l|c|c|c|}
\hline Outcome & HR & $\mathbf{9 5 \% ~ C l}$ & p value \\
\hline Hospitalization for BPH (non surgical reasons) & 0.87 & $0.73-1.05$ & 0.1377 \\
\hline Hospitalization for BPH-related surgery & 0.82 & $0.73-0.93$ & 0.0025 \\
\hline Newly detected prostate cancer & 0.76 & $0.65-0.85$ & 0.0116 \\
\hline
\end{tabular}




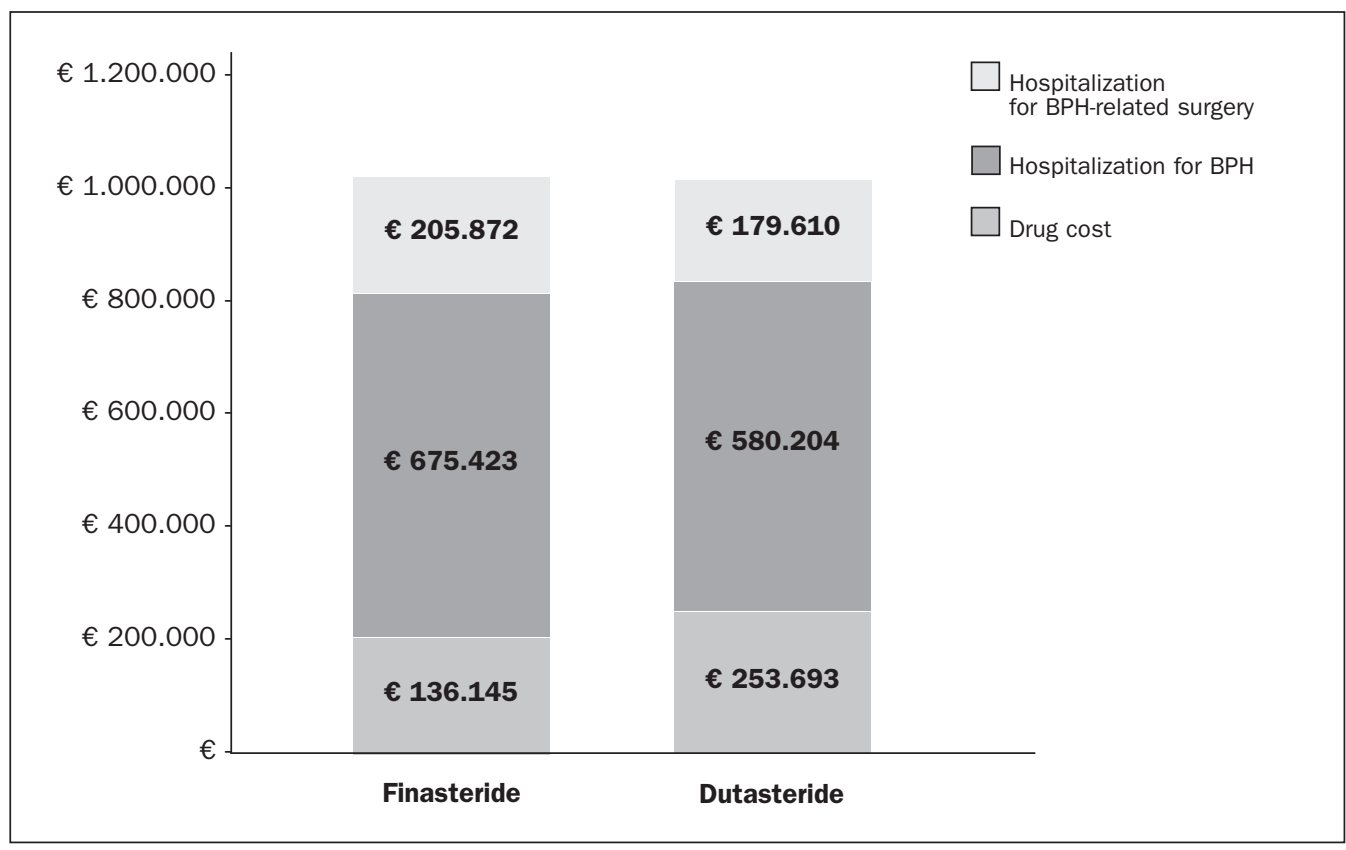

Figure 1.

Dutasteride vs finasteride: comparison of NHS costs for one year treatment of $1000 \mathrm{BPH}$ patient. impact on NHS budget of $10.103 .507 €$ : $25 \%$ of this cost is related to dutasteride cost (253.693 €), $57,2 \%$ is related to hospitalizations for BPH-related surgery (580.204 €) and $17,7 \%$ is related to hospitalizations for BPH-non surgical reasons (179.610 €) (Figure 1).

The full switch from finasteride to dutasteride in an hypothetical cohort of $1000 \mathrm{BPH}$-treated patients for one year generates a net saving of $3.933 €$ to the NHS annual budget.

\section{Discussion}

Dutasteride and finasteride are the two currently available 5ARIs, and are widely recommended in patients with moderate-to-severe BPH-related LUTS (2, 4-6). Large-scale clinical trials have demonstrated that dihydrotestosterone (DHT) suppression with 5ARIs is effective in the treatment of $\mathrm{BPH}$ and might have a role in the prevention of $\mathrm{PCa}(4,5,13,14)$. Previous studies confirmed that dutasteride consistently induces a near-maximal suppression of both serum and intraprostatic DHT in men with BPH and those with PCa (7). Even if the two available 5-ARIs are considered to be virtually equivalent regarding the clinical outcomes $(13,14)$, unfortunately, a direct comparison of the two drugs evaluating the long term effects is still lacking. The EPICS study, the only randomized clinical trial comparing dutasteride vs. finasteride, did not show significant differences between the drugs in terms of clinical efficacy. However, as pointed out by the authors, given the long-term, progressive nature of BPH, the one-year duration of EPICS may limit the potential to observe major differences between dutasteride and finasteride treatment (11).

In lack of relevant, prospective comparative studies, the purpose of this record-linkage study was to analyze the clinical effect of dutasteride and finasteride on BPH-relat- ed hospitalizations and on PCa diagnosis and the economical impact on NHS budget in an Italian cohort.

After the propensity score matching Cox model, the multivariate analysis showed that dutasteride was associated with a statistically significant lower likelihood of hospitalization for BPH-related surgery (Table 4).

These findings are in line with our previous study (12) and the reports from Issa (10) and Fenter (28.

The results of the pharmacoeconomic analysis support health decision maker in the choice of whether or not to implement the treatment of $\mathrm{BPH}$ patient with dutasteride instead of less costly finasteride.

In two papers Fenter and Naslund (28-29) made a real world economic evaluation of dutasteride vs. finasteride for the treatment of BPH patient analyzing restrictively medical and pharmacy claims in two large US administrative databases.

These studies were based on American Medicare-aged population and showed that dutasteride-therapy resulted in less medical costs than finasteride, suggesting that the higher price of dutasteride may be offset by decreased medical resource consumption. In our analysis we also estimated the cost consequence for the Italian NHS of the use of dutasteride instead of finasteride in a hypothetical cohort of $1000 \mathrm{BPH}$-treated patient for one year starting from the clinical differences in major outcomes (hospitalization for surgical and non surgical reason). As a results of our analysis, even in a different NHS framework, the net budget impact of the use of dutasteride instead of finasteride is slightly in favor of dutasteride with a total annual saving of $3.933 €$.

This overall cost saving for men taking dutasteride could create a overall cost advantage for dutasteride despite its higher price. There is also significant additional value to patients who have a lower risk of BPH progression and than prostate surgery under dutasteride, although the 
monetary value of these benefits is difficult to measure and quantify.

As far as the new diagnosis of PCa is concerned, we found a PCa incidence lower in dutasteride- vs. finasteride-treated patients. Although our previous study showed only a positive trend in dutasteride group without a statistical significance, however, in the current study, the wider cohort allowed to reach a statistically significant difference in reduction of PCa diagnosis (HR: $0.76,95 \%$ CI, 0.65-0.85; p = 0.0116) (Table 4).

All these evidence suggest that the clinical benefit of the dual 5a-reductase-isoenzymes inhibition might be slightly better. The two molecules are effective in $\mathrm{BPH}$; nevertheless, due to its peculiar pharmacokynetic and pharmacodynamic characteristics (longer half-life and dual inhibition of 5a-reductase-isoenzymes), dutasteride seems to be more active.

Although our results suggest that there are differences between the two 5ARIs in terms of clinical and economic outcomes, interpretation of the results is limited by the retrospective, non-randomized nature of the study. Moreover, no information about symptomatic burden of the disease, urodynamic parameters, baseline PSA values, number and kind of core biopsies and Gleason score were available in our database. This is a main limitation of the study that hinders any inference about specific outcomes. However, the administrative database are widely used with all the inherent limitations and are considered a valuable source of clinical information (19-21). Moreover, the pharmacoeconomic analysis contains further limitations. Firstly, in clinical practice physician preferences based on clinical characteristics can impact treatment selection which mathematical model can not account for. Secondly, our results are specific to Italy and are driven by local practice and healthcare costs and prices.

\section{Conclusions}

In conclusion, our results suggest slight differences in clinical and economic outcomes between dutasterideand finasteride-treated patients. Further clinical trials are warranted in order to confirm these results and to evaluate the long term effectiveness of these drugs.

\section{ACKNOWLEDGMENTS}

This study was financially supported by an unconditional grant from GlaxoSmithKline.

\section{REFEREnCES}

1. Füllhase C, Chapple C, Cornu JN, et al. Systematic review of combination drug therapy for non-neurogenic male lower urinary tract symptoms. Eur Urol. 2013; 64:228.

2. Oelke M, Bachmann A, Descazeaud A, et al. EAU guidelines on the treatment and follow-up of non-neurogenic male lower urinary tract symptoms including benign prostatic obstruction. Eur Urol. 2013; 64:118.

3. Boyle P, Roehrborn C, Harkaway R, et al. 5-alpha reductase inhibition provides superior benefits to alpha blockade by preventing AUR and BPH-related surgery. Eur Urol. 2004; 45:620.
4. McConnell JD, Roehrborn CG, Bautista O, et al. Medical therapy of prostatic symptoms (MTOPS) research group. The long-term effect of doxazosin, finasteride, and combination therapy on the clinical progression of benign prostatic hyperplasia. N Engl J Med 2003; $349: 2387$

5. Roehrborn CG, Siami P, Barkin J, et al. The effects of combination therapy with dutasteride and tamsulosin on clinical outcomes in men with symptomatic benign prostatic hyperplasia: 4-year results from the CombAT study. Eur Urol. 2010; 57:123

6. Robert G, Descazeaud A, de la Taille A. Lower urinary tract symptoms suggestive of benign prostatic hyperplasia: who are the high-risk patients and what are the best treatment options? Curr Opin Urol. 2011; 21:42.

7. Clark RV, Hermann DJ, Cunningham GR, et al. Marked suppression of dihydrotestosterone in men with benign prostatic hyperplasia by dutasteride, a dual 5 alpha-reductase inhibitor. J Clin Endocrinol Metab 2004; 89:2179.

8. Naslund M, Black L, Eaddy M, Batiste LR. Differences in alpha blocker usage among enlarged prostate patients receiving combination therapy with 5 ARIs. Am J Manag Care. 12007; 3:S17.

9. Naslund M, Regan TS, Ong C, Hogue SL. 5-Alpha reductase inhibitors in men with an enlarged prostate: an evaluation of outcomes and therapeutic alternatives. Am J Manag Care. 2008; 14:S148.

10. Issa MM, Runken MC, Grogg AL, Shah MB. A large retrospective analysis of acute urinary retention and prostate-related surgery in BPH patients treated with 5-alpha reductase inhibitors: dutasteride versus finasteride. Am J Manag Care. 2007; 13:S10.

11. Nickel JC, Gilling P, Tammela TL, et al. Comparison of dutasteride and finasteride for treating benign prostatic hyperplasia: Enlarged Prostate International Comparator Study (EPICS). BJU Int. 2011; 108:388.

12. Cindolo L, Fanizza C, Romero M, et al. The effects of dutasteride and finasteride on BPH-related hospitalization, surgery and prostate cancer diagnosis: a record-linkage analysis. World J Urol. 2013; 31:665.

13. Thompson IM, Goodman PJ, Tangen CM, et al. The influence of finasteride on the development of prostate cancer. N Engl J Med. 2003; 349:215.

14 Andriole GL, Bostwick DG, Brawley OW, et al. Effect of dutasteride on the risk of prostate cancer. N Engl J Med. 2010; 362:1192.

15. Theoret MR, Ning YM, Zhang JJ, et al. The risks and benefits of $5 \alpha$-reductase inhibitors for prostate-cancer prevention. N Engl J Med. 2011; 365:97.

16. Cohen SA, Parsons JK. Combination pharmacological therapies for the management of benign prostatic hyperplasia. Drugs Aging. 2012; $29: 275$

17. WHO Collaborating Centre for Drug Statistics Methodology. ATC Index with DDDs. Oslo, Norway: WHO; 2003

18. US Centers for Disease Control and Prevention. International Classification of Diseases, Ninth Revision, Clinical Modification (ICD-9-CM). http://www.cdc.gov/nchs/icd/icd9cm.htm. Accessibility verified 14 August 2013.

19. Monte S, Macchia A, Pellegrini F, et al. Antithrombotic treatment is strongly underused despite reducing overall mortality among high-risk elderly patients hospitalized with atrial fibrillation. Eur Heart J 2006; 27:2217.

20. Macchia A, Monte S, Romero M, et al. The prognostic influence of chronic obstructive pulmonary disease in patients hospitalized for chronic heart failure. Eur J Heart Fail. 2007; 9:942. 
21. Macchia A, Monte S, Pellegrini F, et al. Depression worsens outcomes in elderly patients with heart failure: an analysis of 48,117 patients in a community setting. Eur J Heart Fail. 2008; 10:714.

22. Charlson ME, Pompei P, Ales KL, MacKenzie CR. A new method of classifying prognostic comorbidity in longitudinal studies: development and validation. J Chronic Dis. 1987; 40:373.

23. Romano PS, Roos LL, Jollis JG. Adapting a clinical comorbidity index for use with ICD-9-CM administrative data: differing perspectives. J Clin Epidemiol. 1993; 46:1075.

24. D'Agostino RB Jr. Propensity score methods for bias reduction in the comparison of a treatment to a non-randomized control group. Stat Med. 1998; 17:2265.

25. Yanovitzky I, Zanutto E, Hornik R. Estimating causal effects of public health education campaigns using propensity score methodology. Eval Program Plann. 2005; 28:209.
26. Parsons LS. Reducing bias in a propensity score matched pair sample using greedy matching techniques. In: Proceedings of the Twenty-Sixth Annual SAS Users Group International Conference. 2004, SAS Institute, Cary, NC

27. Austin PC, Grootendorst P, Anderson GM. A comparison of the ability of different propensity score models to balance measured variables between treated and untreated subjects: a Monte Carlo study. Stat Med. 2007; 26:734.

28. Fenter TC, Runken MC, Black L, Eaddy M. Finasteride versus dutasteride: a real-world economic evaluation. Am J Manag Care. 2007; 13:S23.

29. Naslund M, Eaddy MT, Kruep EJ, Hogue SL. Cost comparison of finasteride and dutasteride for enlarged prostate in a managed care setting among Medicare-aged men. Am J Manag Care. 2008; 14:S167.

\section{Correspondence}

Luca Cindolo, MD, FEBU (Corresponding Author)

lucacindolo@virgilio.it

Francesco.Berardinelli, MD

berardinelli.francesco@gmail.com

Fabiola Raffaella Tamburro, MD

fabiola.tamburro@libero.it

Fabrizio.Pellegrini, MD

fabriziopellegrini85@hotmail.it

Fabio Neri, MD

info@fabioneri.eu

Luigi Schips, MD

luigischips@hotmail.com

S. Pio da Pietrelcina Hospital, Dept. of Urology, Vasto, Italy

Caterina Fanizza, MD

fanizza@negrisud.it

Marilena Romero, MD

romero@negrisud.it

Luisella.Pirozzi, MD

pirozzi@negrisud.it

Department of Clinical Pharmacology and

Epidemiology-Consorzio Mario Negri Sud, Santa Maria Imbaro, Italy

Andrea Pitrelli, MD

andrea.n.pitrelli@gsk.com

Access to Medicine, GlaxoSmithKline spa, Verona, Italy 